\title{
Metodología, temas y disciplinas en la investigación actual sobre migración internacional
}

\author{
Jose Navarro-Conticello \\ Doctorando en la Universidad de Talca, Chile \\ josenavarro83@gmail.com \\ Emilio Moyano-Díaz \\ Profesor Doctor de la Universidad de Talca, Chile \\ emoyano@utalca.cl
}

Resumen La migración internacional es un área de creciente interés científico y político en el mundo, pero se desconoce el volumen y las características de los artículos publicados en bases de datos internacionales. Se busca aquí identificar los enfoques metodológicos predominantes, tipo de problemas, alcance, temas y disciplinas características de la producción científica actual en el área. Un total de 193 artículos indizados en Web of Science de 2012 a 2016 que incluyen en sus títulos el término ‘international migration' son analizados mediante cinco criterios metodológicos de clasificación: paradigma, tipo de problema, alcance, tema y disciplina. Se observa asociaciones significativas relativas a una producción en el área principalmente cuantitativa $\left(\chi^{2}=70,145 \mathrm{gl}(2)\right.$, $\mathrm{p}<.000)$, empírica $\left(\chi^{2}=267,746 \mathrm{gl}(3), \mathrm{p}<.000\right)$, descriptiva y correlacional $\left(\chi^{2}=69,819\right.$ gl $\left.(3), \mathrm{p}<.000\right)$, destacando en cantidad de artículos y alcance de las investigaciones la ciencia económica y la demografía $\left(\chi^{2}=154,922 \mathrm{gl}(8), \mathrm{p}<.000\right)$ entre las disciplinas participantes.

Palabras clave: Migración internacional, cienciometria, métodos, metodología.

\section{Introducción}

La migración humana tiene orígenes prehistóricos, pero sus alcances y consecuencias comenzaron a ser establecidos durante la Antigüedad clásica. La importancia de la Grecia antigua en la historia de la humanidad tiene mucho que ver con la movilidad y los intercambios entre culturas y cosmovisiones alternas. Con la consolidación 
de las polis griegas, hacia el siglo VI a.C., se produjo también la primera delimitación política del migrante en tanto un residente de origen foráneo que es sujeto de derechos y obligaciones en la sociedad que lo acoge. Esa figura, esencialmente legal y administrativa (Sosin, 2016), pero con importantes corolarios socioculturales (Benéitez, 2005; Wijma, 2014), era designada con el término métoikos, que es traducido al español y al portugués como meteco, al inglés como metic, y etimológicamente remite a aquel individuo que cambia de residencia (Liddell; Scott, 1883).

En cambio, la migración como objeto de estudio formal por parte de la academia tiene una historia más breve. El primer artículo científico sobre el tema fue elaborado por el geógrafo alemán Ernst Georg Ravenstein (1885) y tuvo como objetivo formular leyes que pudieran explicar los flujos de personas entre poblados al interior de las naciones. A lo largo del siglo XX el interés académico fue virando gradualmente desde las dinámicas intraestatales hacia las internacionales, que pasaron a concentrar el grueso de las investigaciones. En la actualidad este segundo tipo de estudios ha alcanzado tal predominio que el término genérico 'migración' es utilizado en muchos casos como sinónimo de 'migración internacional' (King; Skeldon, 2010).

El desplazamiento de lo local a lo global en el estudio de la migración se explica por los cambios económicos, demográficos, políticos y socioculturales que tuvieron lugar como consecuencia de la globalización. Aunque a la fecha actual hay menos migrantes internacionales que internos (232 y 740 millones respectivamente) (International Organization for Migration, 2015), las dinámicas de estos últimos no han variado sustantivamente en las últimas décadas, mientras que la migración internacional se ha complejizado y diversificado. El mapa económico mundial se encuentra dividido en un norte y un sur globales (Pal, 2005), profundamente asimétricos pero altamente interconectados mediante las tecnologías de la información y la comunicación, por lo cual millones de personas que viven en las naciones menos poderosas se ven tentadas a emigrar hacia los países más ricos (Portes; DeWind, 2004). Los procesos de reforzamiento y borrosidad de fronteras fisicas, simbólicas y culturales son comunes a la historia de la humanidad (Alonso-Meneses, 2016), pero adquieren nuevas formas en un contexto que se caracteriza por la liquidez de los intercambios y las continuidades entre culturas, pero también por la asimetría y la fragmentación (Bauman, 1999; Beck, 1999; Castells, 2002; Sassen, 1998). A su vez, en distintas partes del orbe surgen debates migratorios altamente mediatizados (Estados Unidos, México y Chile son tres casos resonantes en el continente americano) y crecen los desplazamientos de personas forzados por guerras, desastres naturales o el crimen organizado. Se comprende, entonces, por qué algunos autores definen esta época como la 'era de la migración' internacional (Castles; Miller, 2009).

Tal vez en parte debido al carácter polisémico de su objeto, existe una gran variedad temática, metodológica y disciplinar al interior de los estudios sobre migración internacional. Estos se hallan integrados por disciplinas tan diversas como la economía, la geografia, la psicología y la medicina. Sus temas incluyen las intersecciones entre migración y diáspora (Boyd, 1989; Safran, 1991; Clifford, 1992, 1994; Cohen, 1995, 1997; 
Vertovec; Cohen, 1999; Shuval, 2000; Alonso; Oiarzabal, 2010; Amrith, 2011; Collier, 2013), género (Hondagneu-Sotelo, 1992, 1994; Grieco; Boyd, 1998; Hondagneu-Sotelo; Cranford, 1999; Fouron; Glick-Schiller, 2001; De Jong, 2002; Herrera, 2013; Huang, 2016), raza y etnicidad (Fearon, 2003; Silverstein, 2005; El-Tayeb, 2008; Ford, 2011; Erel; Murji; Nahaboo, 2016), entre muchos otros. Sus métodos, en tanto, van desde abordajes fenomenológicos y situados que buscan comprender la significación de la migración desde la experiencia de los propios migrantes, pasando por aquellos que priorizan la medición y significancia estadística de los flujos y redes migratorios desde una visión macrosocial hasta otros, como los estudios comparados (Izquierdo-Escribano; Cornelius, 2012; Barajas-Escamilla; Solís-Pérez, 2013), que combinan los dos enfoques anteriores.

La diversidad temática, metodológica y disciplinar de los estudios sobre migración ha sido abordada por diversos autores. Algunos se han preocupado por señalar críticamente las fortalezas y debilidades de algunos enfoques metodológicos, produciendo valiosos compendios que pueden servir de guía tanto para estudiosos que comienzan a acercarse al tema como para investigadores entrenados que pretenden incorporar nuevas técnicas de pesquisa (Brettell; Hollifield, 2000; Bonifazi et al., 2008; Iosifides, 2011; Vargas-Silva, 2012; Ariza; Velasco, 2012). Otros, como Baker y Tsuda (2015), han reseñado algunas de las temáticas centrales en este campo del conocimiento.

Sin perjuicio de lo anterior, y como subraya Hugo (2016), hay poca comprensión acerca de cómo el fenómeno es conceptualizado, medido y, más generalmente, estudiado. Se desconoce el peso relativo de diferentes marcos metodológicos, el volumen de producción en distintos temas, tipos de problemas, alcances de los estudios y disciplinas involucradas en la producción académica actual sobre migración internacional y, aparentemente, no se dispone de estudios de base cuantitativa que ayuden a clarificar tales aspectos. En otras palabras, no se cuenta con un diagnóstico actualizado y basado en datos cuantificables que permita conocer qué metodología, temas y disciplinas están prevaleciendo en esa área. El presente trabajo busca responder a este tipo de interrogantes a partir de una revisión cienciométrica o epistemométrica (Krauskopf, 1994) de todos los artículos científicos publicados entre 2012 y 2016 que incluyen el término 'international migration' en su título y se encuentran indizados en las bases de datos de la Colección Principal de Web of Science (WoS). Se trata, entonces, de un estudio de tipo descriptivo, que pretende identificar, cuantificar y caracterizar dichos artículos de acuerdo con cinco criterios - paradigma general, tipo de problema, alcance, tema y disciplina - para ofrecer un panorama de la producción científica actual en el área de la migración internacional.

Por migración internacional se entiende el movimiento de personas que dejan su país de origen o residencia habitual para establecerse permanente o temporalmente en otro país, es decir, cruzando al menos una frontera internacional (International Organization for Migration, 2004). En tanto fenómeno humano, la migración presenta imbricaciones con la cultura, la sociedad y la política que son innegables, involucrando la acción de múltiples actores políticos que se disputan su gestión con diferentes modelos de desarrollo (Skeldon, 2008; de Haas, 2010). Las metodologías no son inmunes a esa discusión; por el contrario, 
han estado tradicionalmente influenciadas por las pugnas por imponer determinados proyectos de sociedad (Cortés, 2015; Castles, 2010). Entendidas como la aplicación de ciertos dispositivos para respondernos preguntas acerca de la realidad, no son neutrales ni dadas naturalmente o de antemano, sino productos culturales que responden al tiempo histórico en el cual se inserta el trabajo investigativo (Moyano, 1999). Lo mismo puede decirse de los temas y las disciplinas involucradas o más relevantes en un momento dado.

Los análisis cienciométricos son útiles para determinar, justamente, la proporción relativa de los diversos temas, metodologías y disciplinas que integran la producción actual - en este caso sobre migración internacional -, ya que mediante ellos se busca cuantificar y extraer significados de bases de datos bibliográficas. Ellos pueden ser practicados con muy variados niveles de desagregación y con diferentes unidades de análisis. Por ejemplo, respecto de cienciometría de temas existe un estudio reciente acerca de felicidad que analiza los artículos publicados en WoS y Scielo en 10 años (MoyanoDíaz, 2016), respecto de la producción en algunas disciplinas y para el caso particular de Chile, se encuentra primeros estudios sobre artículos en revistas chilenas de psicología (Moyano-Díaz; Moyano, 1996; Moyano-Díaz; Ramos, 2000) y en medicina (Krauskopf; Krauskopf, 2008). También hay aquellos en los que se analiza simultáneamente más de una disciplina (Arguimbau-Vivó; Fuentes-Pujol; Gallifa-Calatayud, 2013), y otros que han puesto el acento en la medida y análisis de revistas en ciencias sociales en el mundo y su comparación por países (Pimentel et al., 2016).

La creciente digitalización de las revistas científicas y los artículos publicados en ellas, la accesibilidad de las bases de datos online que los contienen y el avance en los programas informatizados de búsqueda y análisis de información han contribuido a la operatividad de una tarea que antes era realizada con poca frecuencia, medios más básicos y grandes requerimientos de tiempo y recursos. Hoy, un enorme volumen de información referente a la publicación académica en distintos ámbitos está disponible, pero se requiere del análisis para aprehender sus significados. Los estudios cienciométricos resultan de utilidad porque, entre otros factores, entregan una caracterización sistemática y cuantitativa de la producción en un ámbito del conocimiento científico que permite, luego, desagregar cualitativamente su contenido. Esto puede ser de alto valor heurístico para mostrar y caracterizar aquello que existe y lo que no hay o escasea, entre otros aportes que pueden inspirar futuras investigaciones.

\section{Método}

El método utilizado consiste en la búsqueda de artículos con el descriptor international migration' en sus títulos, publicados en las bases de datos de la Colección Principal de WoS en los últimos cinco años - 2012-2016 -, su almacenamiento y posterior análisis mediante la aplicación de criterios metodológicos de clasificación. 
Muestra. La muestra corresponde al total de la población, conformada por 193 artículos publicados de 2012 a 2016 con el término ‘international migration' en sus respectivos títulos, indexados en las bases de datos de la de Colección Principal de WoS. Una búsqueda extendida para los últimos 10 años arrojó un total de 328 artículos, de modo que la muestra corresponde al 58,8 \% de la producción total de la última década. A efectos de mayor pureza del análisis y eventuales réplicas, no fueron contabilizados ni analizados los artículos correspondientes a 2017 (se pesquisaron 7 artículos al 1 de julio), dado que aún no termina el corriente año. En una primera búsqueda en WoS se contabilizó 195 artículos, de los cuales hubo dos que no fueron procesados por la dificultad de ubicar el texto completo de uno de ellos y traducirlo de su idioma original (checo) y, en el otro caso, porque en la base solo aparecía su título (mal mecanografiado) sin resumen ni texto completo.

Instrumentos. Fueron aplicados dos instrumentos. El primero, fue una búsqueda exhaustiva en la WoS mediante el descriptor 'international migration' y el comodín ‘ para incluir variaciones del término, y el almacenamiento del total de artículos encontrados en una carpeta.

El segundo, una plantilla de análisis elaborada ad-hoc, constituida por cinco criterios de interés e informaciones relativas a los componentes generales de los artículos - título, resumen, palabras clave, fecha de publicación - para la identificación y clasificación de cada uno. Los cinco criterios de clasificación están inspirados en la propuesta de Moyano (1999), quien señala que la metodología designa tres procesos conexos: i) el estilo de afrontamiento o paradigma general que guía el abordaje de la realidad estudiada, ii) el tipo de problemas y, iii) el modo en que se responde a las preguntas de investigación. Así, los artículos fueron clasificados y analizados según los siguientes criterios: 1) el paradigma general que guía la investigación, con tres opciones posibles: a) cuantitativo, b) cualitativo o c) mixto (Johnson; Onwuegbuzie, 2004); 2) el tipo de problema analizado, con cuatro opciones: a) conceptual, b) metodológico, c) empírico o d) valorativo (Sierra Bravo, 1987) y, 3) el alcance de las preguntas de investigación, también con cuatro opciones: a) exploratorio, b) descriptivo, c) correlacional o d) explicativo (Hernández; Fernández; Baptista, 2014). Siguiendo esta última clasificación, debe entenderse que el alcance va desde un mínimo conocimiento previo acerca de la realidad - exploratorio -, pasando por la descripción, ya sea narrativa o numérica, de la misma - descriptivo -, hasta llegar a la relación de asociación o covariación entre aspectos de la realidad - correlacional -, para, finalmente, alcanzar el nivel epistémico mayor de la explicación, que permite adquirir un sentido de comprensión de lo real. A estos criterios se les agregan uno temático y otro disciplinar, ambos con clasificación abierta, para identificar temas y disciplinas propios de cada uno de los artículos analizados. Los cinco criterios son resumidos a continuación (Tabla 1). 
Tabla 1 - Criterios clasificatorios de artículos con el término 'migración internacional' en su título, publicados en la Colección Principal de WoS de 2012 a 2016

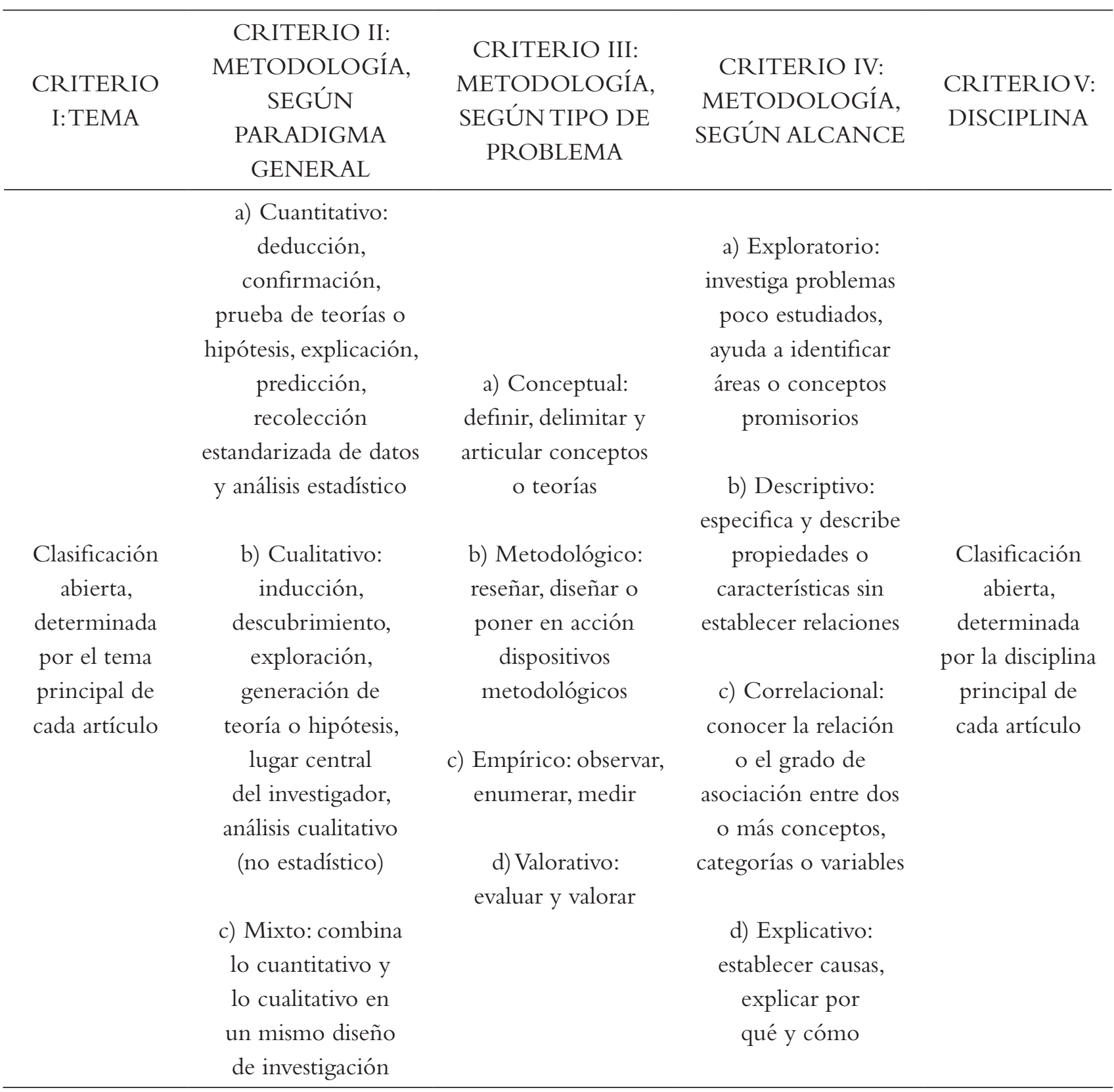

Fuente: elaboración propia a partir de Moyano (1999), Johnson y Onwuegbuzie (2004), Sierra Bravo (1987) y Hernández, Fernández y Baptista (2014).

Procedimiento. Los instrumentos fueron aplicados durante el primer semestre de 2017. Para homogeneizar criterios entre los dos investigadores que clasificaron los artículos se hizo una prueba de coincidencia (porcentaje de acuerdo) entre ambos mediante la aplicación de la plantilla de clasificación a una misma sub-muestra de 30 artículos, con el objetivo de alcanzar al menos un $95 \%$ de acuerdo. En un primer análisis, de 15 artículos, el porcentaje de acuerdo para el conjunto de los cinco criterios clasificatorios fue de 33 $\%$, y de $60 \%$ en cuatro de los cinco criterios. Después de analizar las discrepancias se procedió a analizar los siguientes 15 artículos, llegándose a un $96 \%$ de acuerdo y a partir de allí se procedió a la aplicación definitiva de la plantilla de análisis. Posteriormente, se realizó los cálculos de frecuencia para las categorías clasificatorias de análisis y pruebas de 
significación estadística no paramétricas $\left(\chi^{2}\right)$, según correspondiera, para poder describir los resultados correspondientes a los objetivos del estudio.

\section{Resultados}

En primer lugar, se reporta la frecuencia y porcentaje de artículos según su tipo, siguiendo los criterios metodológicos de paradigma general de investigación, tipo de problema y alcance del estudio. Posteriormente, se realiza un análisis de asociación dentro de cada criterio, a efectos de verificar si existen subtipos dominantes - para descartar que las eventuales diferencias entre subtipos sean simplemente aleatorias - mediante una prueba de asociación o independencia no paramétrica $\left(\chi^{2}\right)$. En tercer lugar, se reporta en qué disciplinas se ubican los estudios acerca de la migración internacional para identificar el peso o contribución de cada una de aquellas relativa al área temática general.

Tabla 2 - Frecuencia, distribución y porcentaje de artículos sobre migración internacional publicados en WoS de 2012 a 2016, según paradigma general, tipo de problema y alcance $(N=193)$

\begin{tabular}{cccccc}
\hline \multicolumn{2}{c}{ PARADIGMA GENERAL } & \multicolumn{2}{c}{ TIPO DE PROBLEMA } & \multicolumn{2}{c}{ ALCANCE } \\
\hline Cuantitativo & $112(58 \%)$ & Empírico & $145(75,1 \%)$ & Exploratorio & $34(17,6 \%)$ \\
Cualitativo & $64(33,2 \%)$ & Conceptual & $33(17,1 \%)$ & Descriptivo & $81(42 \%)$ \\
Mixto & $17(8,8 \%)$ & Metodológico & $9(4,7 \%)$ & Correlacional & $70(36,3 \%)$ \\
& & Valorativo & $6(3,1 \%)$ & Explicativo & $8(4,1 \%)$ \\
\hline
\end{tabular}

Fuente: Elaboración de los autores, 2017.

Se observa (Tabla 2) que el paradigma general dominante es el cuantitativo, que casi dobla al cualitativo en número de artículos. Se trata del predominio del número como medio de descripción de la realidad estudiada, por encima de la palabra o la narración. Por otra parte, los estudios cuyo paradigma general es el mixto son más bien escasos, ya que no alcanzan el $10 \%$ del total dentro de este criterio. Un análisis estadístico permite observar asociaciones significativas relativas a la existencia de un paradigma de investigación principal o predominante en el área, de tipo cuantitativo $\left(\chi^{2}=73,57 \mathrm{gl}(2), \mathrm{p}<.0000\right)$.

Respecto del tipo de problema, se observa un claro predominio de los estudios empíricos, que casi quintuplican los de tipo conceptual - o teóricos -, que les siguen en frecuencia, mientras que los de tipo metodológico y valorativos, aunque existentes, registran una baja frecuencia. Así, se puede afirmar que la mayor parte de los estudios presentan un foco principal en problemas de tipo empírico $\left(\chi^{2}=281,48 \mathrm{gl}(3), \mathrm{p}<.000\right)$,

En lo que respecta a la clasificación según alcance de los estudios, se observa que los artículos de tipo descriptivo y correlacional predominan, distanciándose de las otras dos subcategorías $\left(\chi^{2}=66,80 \mathrm{gl}(3), \mathrm{p}<.000\right)$ y sin diferenciarse significativamente entre ellos.

Por otra parte, acerca de los temas de investigación dentro del ámbito de la migración internacional y su relación con las disciplinas involucradas, se pueden distinguir para el 
conjunto de la muestra analizada 18 temas diferentes de investigación y nueve disciplinas o conjuntos de disciplinas que los tratan (Tabla 3).

Tabla 3 - Frecuencia, distribución y porcentaje de artículos sobre migración internacional publicados en WoS de 2012 a 2016, según tema y disciplina (N=193)

\begin{tabular}{|c|c|c|c|}
\hline \multicolumn{2}{|l|}{ TEMA } & \multicolumn{2}{|c|}{ DISCIPLINA } \\
\hline $\begin{array}{l}\text { Migración internacional, } \\
\text { desarrollo y políticas públicas }\end{array}$ & $25(13 \%)$ & Economía & $58(30,1 \%)$ \\
\hline Flujos y redes migratorias & $23(11,9 \%)$ & Demografia & $54(28 \%)$ \\
\hline Teorías de la migración internacional & $22(11,4 \%)$ & Sociología o Psicología & $23(11,9 \%)$ \\
\hline $\begin{array}{c}\text { Distribución espacial de la } \\
\text { migración internacional }\end{array}$ & $15(7,8 \%)$ & Ciencia Política & $17(8,8 \%)$ \\
\hline Migración internacional y educación & $11(5,7 \%)$ & Medicina & $12(6,2 \%)$ \\
\hline Migración internacional y trabajo & $11(5,7 \%)$ & $\begin{array}{c}\text { Trabajo Social } \\
\text { o Religión }\end{array}$ & $9(4,7 \%)$ \\
\hline Migración internacional y migración interna & $10(5,2 \%)$ & Ciencias Jurídicas & $8(4,1 \%)$ \\
\hline Migración calificada & $10(5,2 \%)$ & $\begin{array}{c}\text { Ciencias de } \\
\text { la Educación, } \\
\text { Comunicación } \\
\text { o Filosofía }\end{array}$ & $7(3,6 \%)$ \\
\hline $\begin{array}{l}\text { Métodos para el estudio de la } \\
\text { migración internacional }\end{array}$ & $9(4,7 \%)$ & $\begin{array}{c}\text { Geografia, Ecología } \\
\text { o Historia }\end{array}$ & $5(2,6 \%)$ \\
\hline $\begin{array}{l}\text { Aspectos psicosociales de la } \\
\text { migración internacional }\end{array}$ & $8(4,1 \%)$ & & \\
\hline Migración internacional y género & $8(4,1 \%)$ & & \\
\hline Migración internacional y remesas & $8(4,1 \%)$ & & \\
\hline Migración internacional y medioambiente & $7(3,6 \%)$ & & \\
\hline Migración internacional y salud & $7(3,6 \%)$ & & \\
\hline Migración internacional y ciclos vitales & $6(3,1 \%)$ & & \\
\hline $\begin{array}{l}\text { Migración internacional y } \\
\text { derechos humanos }\end{array}$ & $6(3,1 \%)$ & & \\
\hline Migración internacional y religión & $4(2,1 \%)$ & & \\
\hline Migración internacional, etnicidad y raza & $3(1,6 \%)$ & & \\
\hline
\end{tabular}

Fuente: Elaboración de los autores, 2017.

Los artículos analizados tratan de una gran variedad de temas de la migración internacional, que han sido agrupados aquí en 18. Entre éstos predominan la migración internacional vinculada al desarrollo y las políticas públicas $(13 \%)$, los flujos y redes migratorias (12\%) y el debate teórico (11\%). Respecto de las disciplinas en las que se enmarcan o ubican, la economía y la demografía son las que congregan la mayor parte de los artículos de la muestra, sumando entre ambas un 58\% del total y sin distanciarse mucho entre sí (solo 3\%). Se trata, entonces, de dos disciplinas claramente dominantes 
desde el punto de vista de su participación en cuanto a número de estudios publicados $\left(\chi^{2}=202,300 \mathrm{gl}(9), \mathrm{p}<000\right)$.

Si al total de estudios de la economía y la demografia se agrega aquellos de la sociología y la psicología se llega al 71 \% del total de los trabajos publicados. A su vez, las disciplinas que menos artículos aportan al tema - inferior a dos dígitos cada una corresponden, en orden decreciente, al trabajo social y la religión, las ciencias jurídicas, la geografia, la ecología y la historia.

Un análisis que combina las frecuencias de artículos en cada disciplina y su distribución por alcance o tipo de estudios se presenta a continuación (Tabla 4).

Tabla 4 - Tabla de contingencia: disciplina y alcance de artículos sobre migración internacional publicados en WoS de 2012 a 2016

\begin{tabular}{|c|c|c|c|c|c|}
\hline \multirow[t]{2}{*}{ DISCIPLINA } & \multicolumn{4}{|c|}{ ALCANCE DEL ESTUDIO } & \multirow[b]{2}{*}{ Total } \\
\hline & Exploratorio & Descriptivo & Correlacional & Explicativo & \\
\hline Economía & 4 & 19 & 33 & 2 & 58 \\
\hline Demografía & 10 & 30 & 10 & 4 & 54 \\
\hline Sociología o Psicología & 7 & 10 & 6 & 0 & 23 \\
\hline Ciencia Política & 3 & 8 & 6 & 0 & 17 \\
\hline Medicina & 2 & 2 & 8 & 0 & 12 \\
\hline Trabajo Social o Religión & 4 & 4 & 1 & 0 & 9 \\
\hline Ciencias Jurídicas & 3 & 3 & 1 & 1 & 8 \\
\hline $\begin{array}{l}\text { Cs. de la Educación, } \\
\text { Comunicación o Filosofia }\end{array}$ & 0 & 3 & 4 & 0 & 7 \\
\hline Geografía, Ecología o Historia & 1 & 2 & 1 & 1 & 5 \\
\hline Total & 34 & 81 & 70 & 8 & 193 \\
\hline
\end{tabular}

Fuente: Elaboración de los autores, 2017.

Se observa que hay dos disciplinas - economía y demografia -, que concentran la mayoría de los estudios y cuyas más altas frecuencias corresponden a estudios de alcance correlacional y descriptivo, respectivamente. Economía y demografía no difieren sustantivamente en la suma total de producción de artículos para el periodo referido, pero en la primera se observan significativamente más estudios de tipo correlacional por sobre las restantes categorías y respecto de la segunda. En medicina - y aunque de mucho menor producción total en este tema -, a su vez, se registra que dos tercios de sus estudios son también de tipo correlacional.

\section{Discusión y conclusiones}

El método cienciométrico (o epistemométrico) que se aplicó al análisis de la producción científica indizada en las bases de datos de WoS durante los últimos cinco años ha mostrado que los temas principales tratados bajo el término 'international migration' 
corresponden a la relación de la migración internacional con el desarrollo y las políticas públicas, en primer lugar, y con los flujos y redes migratorias, en segundo término, concentrando ambas líneas temáticas aproximadamente un cuarto de la producción total en el período. Se encuentran, en cambio, escasos trabajos sobre las vinculaciones entre la migración internacional y el género, la etnicidad, la raza o la religión, los que generalmente provienen de disciplinas como la antropología o los estudios culturales, que no aparecen representadas cuantitativamente de modo significativo en la producción de artículos sobre el tema indizados en WoS.

En contraste con lo anterior, las dos disciplinas más representadas son la economía (30 \%) y la demografía (28\%), seguidas a más distancia por la sociología y la psicología (12 $\%)$ y la ciencia política (9\%). Las dos primeras son disciplinas que tienen la particularidad de que sus estudios son realizados a nivel macrosocial, y cuya materia prima está constituida frecuentemente por grandes bases de datos y así por grandes muestras, locales o internacionales. Es consistente con la definición de demografía que ella se ubique aquí en un lugar muy destacado - segundo y próximo al primero - en la producción de artículos. En efecto, ella tiene como objeto el estudio estadístico de la estructura y la dinámica de las poblaciones y los procesos que determinan su formación, conservación y desaparición, entre los cuales están la emigración e inmigración.

Desde el punto de vista del alcance o los tipos de investigación, economía y medicina comparten aquí también que su tipo de estudios más frecuente es el correlacional. Esto probablemente esté relacionado con el hecho de que su forma de aproximación típica o general es el paradigma cuantitativo, por una parte, y dónde el uso del análisis correlacional es bastante característico independientemente del tema particular de que se trate.Además, ambas pueden utilizar - y lo hacen - bases de datos ya cuantificados o disponibles en las cuentas nacionales, por ejemplo, ingreso por sectores poblacionales o consultas médicas en el sistema público de salud o cifras de morbimortalidad (respectivamente), lo que facilita enormemente la realización de estudios de tipo correlacional.

Respecto al criterio tipo de estudios, para el conjunto de la muestra prevalecen los estudios empíricos $(75 \%)$ por sobre las restantes categorías - conceptual, metodológico, valorativo -, y en economía y demografia esta relación es aún más marcada. Al disponer estas disciplinas de grandes bases de datos, los estudios de tipo empírico derivan con naturalidad en ellas como forma de producir conocimiento. Puede resultar de interés en futuros estudios analizar si sus fuentes principales de datos son primarias o secundarias.

Respecto del alcance hay sólo un 4,7\% de estudios de tipo explicativo que no sólo exploran, describen o correlacionan, sino que se busca propiamente explicar o comprender fenómenos complejos en el área. Casi un 20\% son estudios exploratorios, es decir, en los cuales se intenta abrir nuevos caminos de investigación en el ámbito.

Estas cifras son consistentes con el hecho de que lo más difícil - en cualquier ciencia - es adquirir ese sentido de comprensión de lo real que se espera que provean los estudios de tipo explicativo. Por otra parte, una vez que los investigadores eventualmente alcanzan ese sentido de comprensión del fenómeno en estudio y, como es propio de la espiral del 
conocimiento científico, se reinicia el ciclo con nuevas preguntas o búsqueda de nuevos caminos o innovaciones en sus formas de indagación, dando lugar a la realización de nuevos estudios exploratorios alimentando esta espiral sin fin.

Como fue señalado, el alcance de la investigación va desde lo que se considera en ciencia más básico o inicial - lo exploratorio, cuando recién se comienza a indagar acerca de un tema hasta ese momento poco conocido -, pasando por la descripción - mayor alcance - y la asociación - aún mayor - hasta la explicación, con la cual se pretende establecer las causas de los fenómenos, explicar por qué y cómo es que ocurren. Esto último es el fin último o por excelencia de la ciencia, adquirir o proveer comprensión acerca de la realidad, para lo cual, por cierto, aquella comienza en los estadios iniciales de un nuevo tema o problema para explorarlo, y así, paulatina o gradual y posteriormente, describirlo y construir hipótesis para ello, así como para el análisis de su asociación con otros fenómenos y, final y eventualmente, adquirir comprensión (la cual generalmente no es completa ni final). De este modo, la jerarquía de conocimiento derivable de esta clasificación de estudios no significa que el conocimiento proveniente de la exploración sea de menor valor que el proveniente de los explicativos, ya que sin duda aquel está respondiendo al estadio inicial del conocimiento existente en un tema o a innovación y, así, se trata del 'mejor' conocimiento del que se dispone en ese momento del proceso de producción científica en el tema en cuestión, y, por demás, punto de partida recomendable del camino hacia la comprensión de lo real.

Como se observa aquí, la economía y demografía son las ciencias o disciplinas que más contribuyen a la producción total en el tema, y el nivel de sus estudios - al considerar el criterio alcance - corresponde también a los de tipo correlacional en mayor proporción que las demás ciencias o disciplinas, cuyas modas corresponden a los exploratorios.

Sólo en un 9\% de los trabajos de la muestra se utiliza una metodología mixta, es decir, se combina expresamente ambos paradigmas de investigación en la línea de la integración de lo cuantitativo y lo cualitativo sugerida para los métodos en ciencias sociales en general por Moyano (1999) y Johnson y Onwuegbuzie (2004). Por cierto, esto puede ser vinculado a la relación entre las tradiciones epistemológicas positivista y fenomenológica en ciencias sociales, que aparecen - y son frecuentemente enseñadas - como mundos excluyentes, encerrados en sí mismos, reproduciendo tempranamente una dicotomía de elección forzada para quien se inicia en la investigación y probablemente incidiendo en parte de la investigación publicada. De este modo, se produce una dificultad para abordar problemas integrando lo cuantitativo con lo cualitativo, lo etic con lo emic, el número con la palabra (Moyano, 1999) y de ello - según se observa aquí - no parecen estar exentos los estudios sobre migración internacional. En el presente análisis de la migración internacional, a pesar de que se detecta una gran variedad intra-temática, ello no se acompaña de un uso de métodos cuantitativos, cualitativos y mixtos en una misma proporción.

En lo conceptual, el presente estudio se ha focalizado en la categoría 'migración internacional' por ser un término menos ambiguo y más preciso entre las variantes disponibles, aun cuando esta opción pueda dar lugar a un sesgo en la delimitación de la 
población estudiada. En ese sentido, es probable que la utilización del término 'migración internacional' por sobre otros sea una característica de cierto tipo de estudios o de determinados campos disciplinares que han surgido como predominantes en el análisis aquí presentado. Esto podría ser considerado una eventual limitación en la búsqueda general de la bibliografía analizada, porque 'migración internacional' no es la única forma de referenciar el movimiento de personas entre países. También son utilizados términos como 'migrantes', 'migración' o distintas conjugaciones del verbo 'migrar'. Sin embargo, se considera aquí que parece necesario abrir el debate sobre la pertinencia de los términos empleados en la academia para definir los procesos mediante los cuales las personas nacidas o domiciliadas en un país se desplazan a otros para continuar allí parte de sus vidas, y en ese sentido el rigor conceptual y terminológico debería ser el primer criterio a tener en cuenta si se desea que la producción científica producida sobre el tema sea fácilmente accesible para el conjunto de la comunidad científica. De lo contrario, la dispersión terminológica podría provocar que ciertas contribuciones perdieran visibilidad e impacto. Cuando el tema principal de la investigación es la migración internacional, se sugiere introducir el término en su título, o bien en el resumen y las palabras claves.

Por otra parte, una limitación de tipo metodológico del presente estudio radica en que en la base de datos utilizada - WoS -, no está indizada la información sobre migración internacional proveniente de una importante cantidad de libros, ensayos, informes gubernamentales - Ministerios o Secretarías de los gobiernos - artículos y columnas en medios de comunicación de masas de regiones - como América Latina donde la producción de conocimiento no necesariamente sigue los canales institucionales estandarizados en Europa y Norteamérica. Esta información, que puede incluir tanto comentarios de opinión como ensayos, análisis de problemas asociados a la migración o cifras al respecto, desde visiones disciplinares tan variadas como la política, el periodismo, los estudios culturales y antropológicos o inclusive el arte y las industrias culturales, parece estar cada vez más presente, lo cual amerita y abre interrogaciones y desafíos para nuevos estudios e investigaciones sobre formas alternativas de construcción de conocimiento.

\section{Agradecimientos:}

El autor principal agradece a CONICYT por haber financiado sus estudios de doctorado en Chile (CONICYT-PFCHA/Doctorado Nacional/2017-Folio N ${ }^{\circ}$ 21170178).

Ambos autores agradecen al Grupo de Investigación en Calidad de Vida y Ambientes Saludables (GICVAS), de la Facultad de Psicología, Universidad de Talca (Chile).

\section{Referencias}

AlOnSO, Andoni; OIARZABAL, Pedro (Ed.). Diasporas in the New Media Age: Identity, Politics, and Community. Reno: University of Nevada Press, 2010. 
ALONSO-MENESES, Guillermo (Coord.). Fronteras simbólico-culturales, étnicas e internacionales: Los efectos en la vida de las gentes y sus sociedades. Tijuana: El Colegio de la Frontera Norte, 2016.

AMRITH, Sunil. Migration and Diaspora in Modern Asia. Cambridge: Cambridge University Press, 2011.

AGUIMBAU-VIVÓ, Llorenç: FUENTES-PUJOL, Eulalia; GALLIFA-CATALAYUD, Mónica. Una década de investigación documental sobre cienciometría en España: análisis de los artículos de la base de datos ISOC (20002009). Revista Española de Documentación Científica, v. 36, n. 2, p. 1-9, 2013.

ARIZA, Marina; VELASCO, Laura (Coord.). Métodos cualitativos y su aplicación empírica. Por los caminos de la investigación sobre migración internacional. Ciudad de México: El Colegio de la Frontera Norte-Instituto de investigaciones sociales-UNAM, 2012.

BAKER, Brenda;TSUDA, Takeyuki (Ed.). Migration and disruptions: toward a unifying theory of ancient and contemporary migrations. Gainesville: University Press of Florida, 2015.

BARAJAS-ESCAMILLA, María del Rosío; SOLÍS-PÉREZ, Marlene (Coord.). Fronteras comparadas: desarrollo, trabajo y migración. México-Estados Unidos/Marruecos-Unión Europea. Tijuana-Ciudad de México: El Colegio de la Frontera Norte-Juan Pablos Editor, 2013.

BAUMAN, Zygmunt. Modernidad líquida. Buenos Aires: Fondo de Cultura Económica, 1999.

BECK, Ulrich. What Is Globalization? Cambridge: Polity Press, 1999.

BENÉITEZ, Benita. La ciudadanía de la democracia ateniense. Foro Interno, v. 5, p. 37-58, 2005.

BONIFAZI, Corrado; OKÓLSKI, Marek; SCHOORL, Jeannette; SIMON, Patrick. International Migration in Europe: New Trends and New Methods of Analysis. Amsterdam: Amsterdam University Press, 2008.

BOYD, Monica. Family and personal networks in international migration: recent developments and new agendas. International Migration Review, v.23, n. 3, p. 638-670, 1989.

BRETTELl, Caroline; HOLlifield, James (Ed.). Migration Theory: Talking Across Disciplines. New York: Routledge, 2000.

CASTELLS, Manuel. La Era de la Información. Vol. I: La Sociedad Red. Ciudad de México: Siglo XXI, 2002.

CASTLES, Stephen. Understanding Global Migration: A Social Transformation Perspective. Journal of Ethnic and Migration Studies, v. 36, n. 10, p. 1565-1586, 2010.

; MILLER, Mark. The Age of Migration: International Population Movements in the Modern World. Basingstoke: Palgrave MacMillan, 2009.

CLIFFORD, James. Travelling Cultures. En: GROSSBERG, Lawrence, NELSON, Cary; TREICHLER, Paula (Ed.). Cultural Studies. New York: Routledge, 1992.

. Diasporas. Cultural Anthropology, v. 9, n. 3, p. 302-338, 1994.

COHEN, Robin. Rethinking 'Babylon': Iconoclastic Conceptions of the Diasporic Experience. New Community, v. 21, n. 1, p. 5-18, 1995.

Global Diasporas: An Introduction. London: University College of London Press, 1997.

COLLIER, Paul. Exodus: Immigration and Multiculturalism in the 21st Century. Oxford: Oxford University Press, 2013.

CORTÉS, Fernando. Desarrollo de la metodología en ciencias sociales en América Latina: posiciones teóricas y proyectos de sociedad. Perfiles Latinoamericanos, v.23, n.45, p. 181-202, 2015.

DE JONG, Gordon. Expectations, Gender, and Norms in Migration Decision-Making. Population Studies, v. 54, p. 307-319, 2002.

DE HAAS, Hein. Migration and Development: A Theoretical Perspective. International Migration Review, v. 44, n. 1, p. 227-264, 2010. 
EL-TAYEB, Fatima. 'The Birth of a European Public': Migration, Postnationality, and Race in the Uniting of Europe. American Quarterly, v. 60, n.3, p.649-670, 2008.

EREL, Umut; MURJI, Karim; NAHABOO, Zaki. Understanding the contemporary race-migration nexus. Ethnic and Racial Studies Review, v.39, p. 1339-1360, 2016.

FEARON, James. Ethnic and Cultural Diversity by Country. Journal of Economic Growth, v.8, p.195-222, 2003.

FORD, Robert. Acceptable and Unacceptable Immigrants: How Opposition to Immigration in Britain Is Affected by Migrants' Region of Origin. Journal of Ethnic and Migration Studies. v.37, n.7, p. 1017-1037, 2011.

FOURON, Georges; GLICK-SCHILLER, Nina. All in the Family: Gender, Transnational Migration, and the Nation-State. Identities, v.7, p. 539-582, 2001.

GRIECO, Elizabeth; BOYD, Monica. Women and Migration: Incorporating Gender Into International Migration Theory. Center for the Study of Population, Florida State University, Working Paper, p. 98-139, 1998.

HERRERA, Gioconda. Gender and International Migration: Contributions and Cross-Fertilizations. Annual Review of Sociology, v. 39, n. 471-489, 2013.

HUANG, Shu-Mei. Can Travelling Mothers Ever Arrive? Articulating Internal and International Migration within a Transnational Perspective of Care. Population, Space and Place, v. 7, p. 705-717, 2016.

HUGO, Graeme. Internal and international migration in East and South East Asia: exploring the linkage. Population, Space and Place, v. 22, p. 651-668, 2016.

HERNÁNDEZ, Roberto; FERNÁNDEZ, Carlos; BAPTISTA, Pilar. Metodología de la investigación. Ciudad de México: McGraw Hill, 2014.

HONDAGNEU-SOTELO, Pierrette. Overcoming Patriarchal Constraints: The Reconstruction of Gender Relations Among Mexican Immigrant Women and Men. Gender and Society, v.6, p. 393-415, 1992.

(Ed.). Gendered Transitions: Mexican Experiences of Immigration. Berkeley: University of California Press, 1994.

; CRANFORD, Cynthia. Gender and Migration. En: CHAFETZ, Janet (Ed.). Handbook of the Sociology of Gender. New York: Kluwer Academic/Plenum Publishers, p. 105-127, 1999.

INTERNATIONAL OR GANIZATION FOR MIGRATION. Glossary on Migration. Ginebra: IOM, 2004.

World Migration Report 2015. Migrants and Cities: New Partnerships to Manage Mobility. Ginebra: IOM, 2015.

IOSIFIDES, Theodoros. Qualitative Methods in Migration Studies: A Critical Realist Perspective. Farnham: Ashgate Publishing, 2011.

IZQUIERDO-ESCRIBANO, Antonio; CORNELIUS, Wayne. Políticas de control migratorio. Estudio comparado de España y Estados Unidos. Barcelona: Bellaterra, 2012.

JOHNSON, Burke; ONWUEGBUZIE, Anthony. Mixed Methods Research:A Research Paradigm Whose Time Has Come. Educational Researcher, v.33, n.7, p. 14-26, 2004.

KING, Russel; SKELDON, Ronald. 'Mind the Gap!' Integrating Approaches to Internal and International Migration. Journal of Ethnic and Migration Studies, v.36, n.10, p. 1619-1646, 2010.

KRAUSKOPF, Manuel. Epistemometría, a term contributing to express the meaning and potential methodologies of scientometrics in spanish speaking countries. Scientrometrics, v.30, n.2-3, p. 425-428, 1994.

; KRAUSKOPF, Erwin. Una mirada epistemométrica de la Revista Médica de Chile y su aporte al conocimiento en Medicina. Revista Médica de Chile, v.136, p. 1065-1072, 2008.

LIDDELL, Henry; SCOTT, Robert. Greek-English Lexicon. Nueva York: Harper \& Brothers-Franklin Square, 1883.

MASSEY, Sean; BARRERAS, Ricardo. Introducing “Impact Validity”. Journal of Social Issues, v. 69, p. 615-632, 2013. 
MOYANO-Díaz, Emilio. Hacia la integración de métodos cualitativos y cuantitativos en la investigación social. Revista de la Facultad de Humanidades de la Universidad de Santiago de Chile, v.4, p. 59-86, 1999.

MOYANO-Díaz, Emilio. Trends and challenges for the research of happiness in Latin América. En: ROJAS, Mariano. Handbook of Happiness Research in Latin América, International Handbooks Quality-of -Life, Dordrecht: Springer. 2016.

; MOYANO, Andrea. Panorama de la investigación publicada en Chile. Interdisciplinaria, Revista de Psicología y Ciencias Afines, v.13, n.2, p.79-95, 1996.

; RAMOS, Nadia. Contexto y evaluación de la investigación psicológica en el Chile de final de siglo. Psykhé, v. 9, n.1, p. 63-75, 2000.

PAL, Sing. Lexicon on Geography of Development. Delhi: Concept Publishing Company, 2005.

PIMENTEL, Gonzalo; GARCÉS, Alejandro; KUZMINSKY, Susan; AGÜERO, Carolina; NÚÑEZ, Lautaro. El conocimiento en disputa. Algunas observaciones sobre cienciometría, sistemas de acceso y ciencia social latinoamericana. Estudios Atacameños, San Pedro de Atacama. v.53, 2016.

PORTES, Alejandro; DEWIND, Josh. A Cross-Atlantic Dialogue: The Progress of Research and Theory in the Study of International Migration, International Migration Review, v. 38, n.3, p. 828-851. 2004.

RAVENSTEIN, Ernst. The Laws of Migration. Journal of the Statistical Society of London, v.48, n.2, p. 167-235, 1885.

SAFRAN, William. Diaspora in Modern Societies: Myths of Homeland and Return. Diaspora, v.1, n.1, p. 83-99, 1991.

SASSEN, Saskia. Globalization and its discontents. Essays on the New Mobility of People and Money. New York: New Press, 1998.

SHUVAL, Judith. Diaspora Migration: Definitional Ambiguities and a Theoretical Paradigm. International Migration, v.38, n.5, p.41-56, 2000.

SIERRA BRAVO, Restituto. La Investigación Social. Teoría y Ejercicios. Madrid: Paraninfo, 1987.

SILVERSTEIN, Paul. Immigrant Racialization and the New Savage Slot: Race, Migration, and Immigration in the New Europe. Annual Review of Anthropology, v.34, p. 363-384, 2005.

SKELDON, Ronald. International Migration as a Tool in Development Policy: A Passing Phase? Population and Development Review, v. 34, n. 1, p. 1-18, 2008.

SOSIN, Joshua. A metic was a metic. Historia, v. 65, n. 1, p. 2-13, 2016.

VARGAS-SILVA, Carlos (Ed.). Handbook of Research Methods in Migration. Cheltenham: Edward Elgar Publishing, 2012.

VERTOVEC, Steven y COHEN, Robin (Ed.). Migration, Diasporas and Transnationalism. Aldershot: Edward Elgar Publishing, 1999.

WEB OF SCIENCE. Web of Knowledge, Thomson Reuters.

WIJMA, Sara. Embracing the Immigrant: The participation of metics in Athenian polis religion (5th-4th century BC). Stuttgart: Franz Steiner Verlag, 2014. 


\title{
Metodologia, temas e disciplinas na investigação atual sobre migração internacional
}

\section{Resumo}

A migração internacional é uma área de crescente interesse científico e político no mundo, mas se desconhece o volume e os tipos de artigos acadêmicos publicados nas bases de dados internacionais. Procura-se identificar aqui os enfoques metodológicos predominantes, tipo de problemas, alcance, temas e disciplinas características da produção científica atual nesse campo de estudos. Um total de 193 artigos indexados na Web of Science de 2012 a 2016 que incluem em seus títulos o termo "international migration" foram analisados usando cinco critérios metodológicos de classificação: paradigma, tipo de problema, alcance, disciplina principal e tema. Observaram-se associações significativas relativas a uma produção na área principalmente quantitativa, $\left(\chi^{2}=70,145 \mathrm{gl}(2), \mathrm{p}<.000\right)$, empírica $\left(\chi^{2}=267,746 \mathrm{gl}(3), \mathrm{p}<.000\right)$, descritiva e correlacional $\left(\chi^{2}=69,819\right.$ gl $\left.(3), \mathrm{p}<.000\right)$, destacando em quantidade e alcance dos estudos a ciência econômica e a demografia $\left(\chi^{2}=154,922 \mathrm{gl}(8), \mathrm{p}<.000\right)$ entre as disciplinas participantes.

Palavras-chave: Migração internacional, cienciometría, métodos, metodologia.

\section{Methodology, themes and disciplines in current research on international migration}

\begin{abstract}
International migration is an area of growing scientific and politic interest worldwide, but both the volume and characteristics of articles published in international databases remain unknown. This paper aims to identify the dominant methodological approaches, type of problems, scope, themes, and disciplines of the current scientific production in this area. A total of 193 articles indexed in the Web of Science from 2012 to 2016 that include in their titles the term "international migration" were analyzed using five methodological criteria of classification: paradigm, type of problem, scope, theme and discipline. Significant associations were observed, relating to a mainly quantitative (X2=70,145 Df. (2), $\mathrm{p}<.000)$, empirical (X2=267,746 Df. (3), $\mathrm{p}<.000$ ), descriptive and correlational (X2=69,819 Df. (3), $\mathrm{p}<.000$ ) production, in which economic science and demography stand out in quantity of articles and scope of their research $(\mathrm{X} 2=154,922$ Df. $(8), \mathrm{p}<.000)$ among the different disciplines.
\end{abstract}

Keywords: International migration, scientometrics, methods, methodology. 